\title{
Increased oxidative phosphorylation in response to acute and chronic DNA damage
}

Lear E Brace ${ }^{1}$, Sarah C Vose ${ }^{2}$, Kristopher Stanya ${ }^{1}$, Rose M Gathungu ${ }^{3}$, Vasant R Marur ${ }^{3}$, Alban Longchamp ${ }^{1}$, Humberto Treviño-Villarreal ${ }^{1}$, Pedro Mejia ${ }^{1}$, Dorathy Vargas ${ }^{1}$, Karen Inouye ${ }^{1}$, Roderick T Bronson ${ }^{4}$, Chih-Hao Lee $^{1}$, Edward Neilan ${ }^{5}$, Bruce S Kristal ${ }^{3}$ and James R Mitchell ${ }^{1}$

Accumulation of DNA damage is intricately linked to aging, aging-related diseases and progeroid syndromes such as Cockayne syndrome (CS). Free radicals from endogenous oxidative energy metabolism can damage DNA, however the potential of acute or chronic DNA damage to modulate cellular and/or organismal energy metabolism remains largely unexplored. We modeled chronic endogenous genotoxic stress using a DNA repair-deficient $\mathrm{Csa}^{-/} \mid \mathrm{Xpa}^{-/-}$mouse model of CS. Exogenous genotoxic stress was modeled in mice in vivo and primary cells in vitro treated with different genotoxins giving rise to diverse spectrums of lesions, including ultraviolet radiation, intrastrand crosslinking agents and ionizing radiation. Both chronic endogenous and acute exogenous genotoxic stress increased mitochondrial fatty acid oxidation (FAO) on the organismal level, manifested by increased oxygen consumption, reduced respiratory exchange ratio, progressive adipose loss and increased FAO in tissues ex vivo. In multiple primary cell types, the metabolic response to different genotoxins manifested as a cell-autonomous increase in oxidative phosphorylation (OXPHOS) subsequent to a transient decline in steady-state NAD+ and ATP levels, and required the DNA damage sensor PARP-1 and energy-sensing kinase AMPK. We conclude that increased FAO/OXPHOS is a general, beneficial, adaptive response to DNA damage on cellular and organismal levels, illustrating a fundamental link between genotoxic stress and energy metabolism driven by the energetic cost of DNA damage. Our study points to therapeutic opportunities to mitigate detrimental effects of DNA damage on primary cells in the context of radio/chemotherapy or progeroid syndromes.

npj Aging and Mechanisms of Disease (2016) 2, 16022; doi:10.1038/npjamd.2016.22; published online 13 October 2016

\section{INTRODUCTION}

The process of generating energy through mitochondrial oxidative phosphorylation (OXPHOS) inevitably results in production of free radicals that can damage cellular macromolecules, including DNA. Consistent with this, interventions that increase OXPHOS (e.g., fasting) can also lead to an increase in free radical generation, at least transiently. ${ }^{1}$ Because of the potential detrimental effects of free radical damage, cells have evolved a variety of mechanisms of detoxification, many of which respond to oxidative stress in an inducible fashion. For example, the transcription factor NRF2 controlling expression of key enzymes in the glutathione pathway is itself activated by oxidative and electrophilic stressors. ${ }^{2}$ On the other hand, because energy generation through aerobic glycolysis rather than OXPHOS could potentially limit further oxidative DNA damage accumulation, ${ }^{3}$ altering metabolism could be another potential strategy to limit free radical damage. As proof of principle, a number of cell types can rapidly shift between OXPHOS and glycolysis depending on environmental cues, e.g., upon antigen stimulation in the case of immune cells, ${ }^{4}$ although the potential of DNA damage to contribute to this process remains untested.

DNA damage can also be energetically costly, e.g., upon activation of the DNA damage response protein, PARP-1. PARP-1 binds directly to single-stranded DNA and generates chains of
poly-ADP-ribose (PAR) in response to breaks that occur directly or indirectly upon ultraviolet radiation (UV), ${ }^{5}$ ionizing radiation $(\mathrm{IR}){ }^{6}$ topoisomerase inhibition $^{7}$ or DNA alkylation. ${ }^{8}$ PARP-1 activation by massive DNA damage leads directly to NAD+ depletion and subsequently to ATP depletion and cell death via necrosis ${ }^{9}$ or apoptosis. ${ }^{10}$ Although this demonstrates the indirect potential of DNA damage to alter cellular energy levels, with profound downstream consequences on cell fate, ${ }^{11}$ the molecular mechanisms underlying PARP-1 induced ATP loss remain unresolved. ${ }^{12}$ One possibility is through depletion of NAD+, a key metabolite in both glycolytic and oxidative energy metabolism.

Although the PARP-1 example serves to illustrate the high energetic cost of excessive DNA damage, how cells respond to perturbations in energy homeostasis induced by sublethal doses of DNA damage remains poorly characterized. One clue is the discovery that genotoxic stressors including UV-C, $I^{13}$ and cisplatin ${ }^{14}$ can activate the AMP-activated protein kinase (AMPK), which acts to restore energy balance by inhibiting ATP-consuming processes and activating processes to generate ATP. ${ }^{15}$ AMPK is regulated allosterically by $\mathrm{AMP}$, but also requires phosphorylation by upstream kinases such as LKB1 and CaMKK ${ }^{15}$ or p53 transcriptional targets, sestrin $1 / 2 .^{16}$ Currently, neither the mechanism by which DNA damage activates AMPK, nor the consequences of such activation, are clearly understood.

\footnotetext{
${ }^{1}$ Department of Genetics and Complex Diseases, Harvard T. H. Chan School of Public Health, Boston, MA, USA; ${ }^{2}$ Division of Environmental Health, Vermont Department of Health, Burlington, VT, USA; ${ }^{3}$ Department of Neurosurgery, Harvard Medical School, Brigham and Women's Hospital, Boston, MA, USA; ${ }^{4}$ Rodent Histopathology Core, Department of Pathology, Harvard Medical School, Boston, MA, USA and ${ }^{5}$ Genetics and Metabolism Division, Boston Children's Hospital, Harvard Medical School, Boston, MA, USA. Correspondence: JR Mitchell (jmitchel@hsph.harvard.edu)

Received 27 August 2015; revised 11 June 2016; accepted 25 July 2016
} 
a

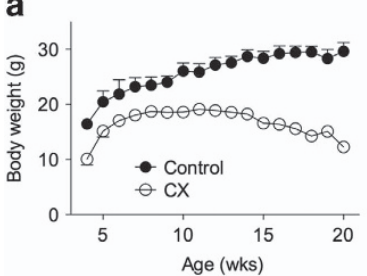

b

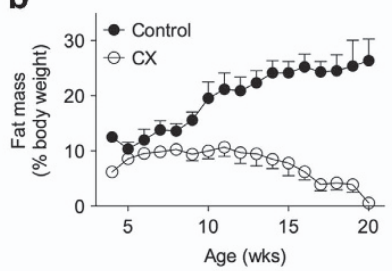

C

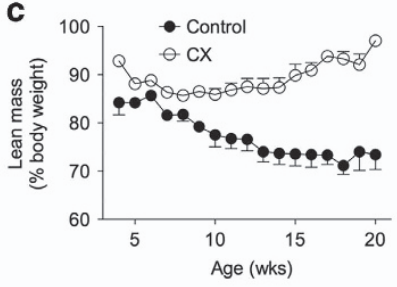

d

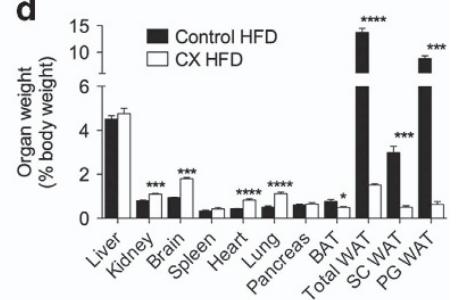

e

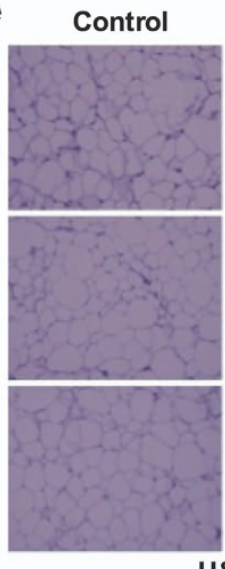

f

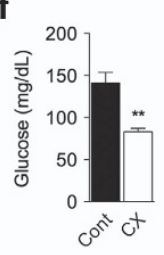

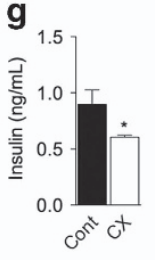

h

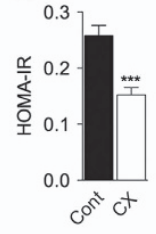

12wks

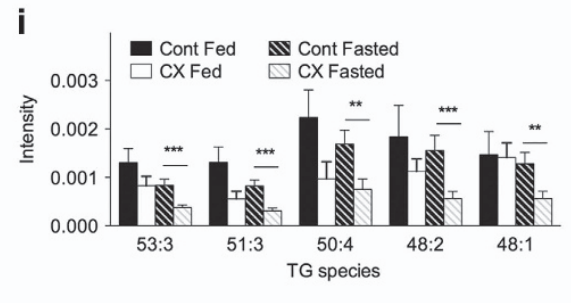

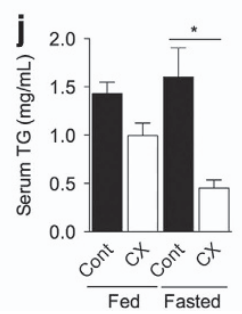

Figure 1. Perturbations in energy metabolism indicative of improved metabolic fitness in CX mice. (a-c) Changes in body weight (a), percentage fat (b) and lean mass (c) over time in CX and control mice ( $n=14-16 /$ group). (d) Organ weights upon harvest of 14 week mice on a high-fat diet (HFD) expressed as a percentage body weight, $n=4 /$ group; Student's $t$-test. (e) Hematoxylin and eosin (H\&E) staining of paraffinembedded perigonadal white adipose tissue (WAT) from control and CX mice. (f-h) Blood glucose (f), serum insulin levels (g) and HOMA-IR (h) of 12-16 week control and CX mice ( $n=7-11 /$ genotype); Student's $t$-test. (i) Relative intensity of the indicated triglyceride (TG) species in serum from fed or fasted CX or control mice by unbiased lipidomics ( $C X=5 /$ group, control $=10 /$ group); two-way analysis of variance (ANOVA) with Bonferroni post-test. (j) Total serum TG in fasted and fed WT and CX mice ( $n=3 /$ group); Student's $t$-test. ${ }^{*} P<0.05$, ${ }^{* *} P<0.01$, $* * * P<0.001, * * * * P<0.0001$.

Thus, although it is clear that the byproducts of normal energy metabolism can cause DNA damage, and that DNA damage can impact cellular energy metabolism, whether and how DNA damage itself exerts feedback control over energy metabolism is poorly characterized, particularly on the organismal level. Here, we set out to directly test the potential of DNA damage to affect energy metabolism, and to identify common adaptive metabolic changes on the cellular and organismal levels in response to both chronic and acute DNA damage.

For chronic damage, we utilized a mouse model of the congenital DNA repair deficiency disorder, Cockayne syndrome (CS). CS is characterized by growth failure, loss of adiposity, neurodegeneration and photosensitivity without prevalence of skin cancer. ${ }^{17}$ It is caused by alterations in proteins including CSA or CSB that share a common function in transcription-coupled nucleotide excision repair (NER) of bulky, helix-distorting lesions, e.g., those caused by UV light that sterically hinder RNA polymerases and block transcription. ${ }^{18}$ Recently, we reported a mouse model of CS lacking both Csa and Xpa, another NER gene, (heretofore referred to as CX) that recapitulates many aspects of the human progeria. ${ }^{19}$ As a model of acute genotoxic stress, we subjected wild-type (WT) mice and cells with genotoxins (UV-C, IR, mitomycin C (MMC) and irinotecan) giving rise to diverse spectrums of DNA lesions. Here, we report that multiple forms of acute and chronic genotoxic stress engender a PARP-1-dependent metabolic switch characterized by increased fatty acid oxidation (FAO) and OXPHOS as an adaptive response to maintain energy homeostasis on both organismal and cellautonomous levels.

\section{RESULTS}

Perturbations in energy metabolism indicative of improved metabolic fitness in CX mice

A number of mouse models with severe symptoms of CS ('NER progeria' in mice), display apparent alterations in systemic metabolism during post-natal development, including loss of adiposity, perturbations of the post-natal GH-IGF-1 axis and hypoglycemia contributing to death before weaning. ${ }^{20}$ Using the CX mouse model that survives weaning with high penetrance, ${ }^{19}$ we first characterized changes in adiposity, glucose and lipid homeostasis as markers of whole-body energy metabolism. Although physically smaller than control (includes WT, CSA single KO and XPA single KO) mice, CX mice increased in body mass during post-natal development at a similar rate. Maximal body mass was attained by 8 weeks and was stable for $\sim 5$ weeks before onset of terminal senescent weight loss over the remainder of the lifespan (Figure 1a). Body fat in CX mice calculated as either a percentage of body weight (Figure 1b) or in absolute grams (Supplementary Figure S1a) paralleled body weight, peaking $\sim 8$ weeks and declining after 13 weeks. Although the absolute lean mass declined with age in CX mice (Supplementary Figure S1b), the percentage lean mass remained stable between 13 and 20 weeks (Figure 1c). CX mice fed a highfat diet (HFD) failed to normalize relative and absolute weights of subcutaneous or perigonadal white adipose tissue (WAT) depots compared with controls (Figure 1d, Supplementary Figure S1c), consistent with the failure of HFD feeding to rescue $C X$ longevity. ${ }^{19}$ Progressive histological investigation of WAT from CX mice showed a decline in mature adipocyte size (Figure 1e), but no visible evidence of crown-like structures indicative of 
inflammatory macrophage activation by hematoxylin and eosin or in WAT from 16 week CX mice as shown by F4/80 immunohistochemistry (Supplementary Figure S1d) or fluorescence-activated cell sorting (FACS) analysis of F4/80+/CD11b+ stromal vascular fraction (Supplementary Figure S1e). These data suggest altered functionality of CX WAT rather than loss of cellularity due to inflammation.

In 14-16 week CX mice, fasted blood glucose and insulin levels were significantly reduced (Figure $1 \mathrm{f}, \mathrm{g}$ ), leading to an improved HOMA-IR (Figure $1 \mathrm{~h}$ ). Insulin challenge trended towards increased glucose disposal in 14 week CX mice (Supplementary Figure S1f), and glucose tolerance tests revealed no difference (Supplementary Figure S1g), suggestive of improved whole-body insulin sensitivity but reduced capacity for stimulated insulin secretion in CX mice, similar to long-lived models such as growth hormone receptor knockout mice. ${ }^{21}$ Circulating leptin levels in the fasted state were significantly decreased in CX versus control mice (Supplementary Figure S1h) but adiponectin levels were unchanged (Supplementary Figure S1i). Analysis of serum lipids using unbiased mass spectrometry revealed significant differences in multiple lipid species in the fasted state and a trend towards a reduction in the fed state in 16 week CX mice relative to both WT and single KO controls, including a number of individual triglyceride species (Figure 1i, Supplementary Figure S1j). Measurement of bulk serum triglyceride in a separate cohort of control and CX mice confirmed a significant reduction in the fasted state (Figure 1j).

Increased FAO in CX mice in vivo and cells in vitro

Reduced adiposity, improved glucose homeostasis and reduced circulating lipids are characteristics of the pro-longevity intervention known as dietary restriction (DR), defined as reduced food intake without malnutrition, and linked an overall metabolic shift to FAO.22 Previously, DR-like phenotypes including improved insulin sensitivity and increased oxidative stress resistance have been observed in CS mouse models. ${ }^{23}$ We used indirect calorimetry to test the hypothesis that FAO was increased in CX mice despite ad libitum access to food. CX mice consumed more $\mathrm{O}_{2}$ (Figure 2a) and produced more $\mathrm{CO}_{2}$ (Figure $2 \mathrm{~b}$ ) than control littermates, but without significant differences in physical activity (Supplementary Figure S2a) or food intake (Supplementary Figure S2b). Importantly, a reduced respiratory exchange ratio (RER), indicative of preferential use of fat or protein relative to carbohydrate, was observed (Figure 2c, Supplementary Figure S2c), but only during the dark phase and despite normal intake of a nutritionally complete diet with $70 \%$ calories in the form of carbohydrates. Ketone bodies were appropriately elevated in both fasted control and CX mice, suggestive of an intact fasting response in CX mice without evidence of malabsorption (Supplementary Figure S2d).

Gene expression profiling of skeletal muscle from fasted CX relative to control mice revealed a significant increase in many FAO-related genes (Figure 2d). Consistently, soleus muscle from CX mice had a significantly elevated capacity to oxidize the radiolabelled $16 \mathrm{C}$-saturated fatty acid palmitate relative to controls (Figure 2e). Interestingly, soleus from CSA KO animals with a much milder metabolic phenotype displayed a trend toward increased FAO capacity, whereas soleus from XPA KO mice with no reported metabolic phenotype did not. Ex vivo FAO capacity was also significantly increased in livers of CX mice relative to controls (Figure $2 \mathrm{f}$ ).

Because FAO can be driven by substrate availability in vivo, we next asked if increased FAO capacity was an intrinsic property of CX cells. To this end, primary, low-passage dermal fibroblasts (mouse dermal fibroblasts (MDFs)) were isolated from tails of preweaning heterozygote WT and CX mice. We note that cells from CS patients and related rodent models do not show defects in cellular proliferation or onset of premature cellular senescence, ${ }^{24}$ nor did we observe this in CX cells.

Under standard serum-free Seahorse media conditions with $11 \mathrm{mM}$ glucose, CX MDFs had a higher oxygen consumption rate (OCR) than control cells (Figure 2g), consistent with increased mitochondrial OXPHOS, and slightly but not significantly reduced extracellular acidification rate (ECAR) indicative of aerobic glycolysis (Supplementary Figure S2e). Both measures were normalized to total cellular protein content, a marker of cell viability, which was not different amongst genotypes (Supplementary Figure S2f); mitochondrial content was slightly but not significantly increased in CX MDFs relative to control cells (Supplementary Figure S2g). Under low-glucose conditions $(2.5 \mathrm{mM})$, addition of the fatty acid palmitate increased OCR to a significantly higher level in CX MDFs compared with control cells (Figure 2h). Thus, increased OXPHOS activity appears to be a cellintrinsic property of CX cells independent of the substrate, glucose or fatty acid that is available for oxidation.

Increased FAO is a cell-autonomous, adaptive response triggered by genotoxic stress in CX cells

As increased FAO in CX cells/animals could be due to changes distinct from accumulated unrepaired DNA lesions, e.g., defects in transcriptional regulation associated with some forms of $\mathrm{CS}^{25}$ we next tested the potential of DNA damage to directly impact OXPHOS/FAO capacity. To this end, MDFs were challenged with UV-C to induce bulky DNA lesions without generation of free radicals that can damage lipids and proteins. Twenty-four hours after a relatively low dose of $4 \mathrm{~J} / \mathrm{m}^{2} \mathrm{UV}-\mathrm{C}$ versus mock treatment, OCR was significantly increased as a function of UV-C exposure in CX MDFs under conditions favoring FAO ( $2.5 \mathrm{mM}$ glucose, addition of palmitate; Figure $3 \mathrm{a}$, AUC inset) or glucose oxidation $(11 \mathrm{mM}$ glucose, without palmitate; Figure $3 \mathrm{~b}$ ), consistent with metabolic substrate flexibility as observed above. OCR readings were normalized to total protein content calculated from the same cells immediately after the final reading; protein content was not significantly altered by low-dose UV-C in this time frame (Supplementary Figure S3a). Mitochondrial content normalized to total protein was increased slightly but significantly on UV-C in both cell types (Supplementary Figure S3b), and thus unlikely to be causative of the specific increase in OCR upon UV-C in CX MDFs. ECAR was significantly reduced in UV-C treated CX cells compared with untreated WT cells (Supplementary Figure S3c).

Direct measurements of FAO capacity in dermal fibroblast cultures from CX mice (Figure 3c), as well as CSA and CSB primary human diploid fibroblasts (HDF; Figure 3d), revealed significant increases $24 \mathrm{~h}$ after UV-C irradiation. Increased FAO capacity was not specific to dermal fibroblasts, but was also observed on UV-C irradiation in CX-derived pre-adipocytes (Supplementary Figure S3d). Consistent with the potential for increased FAO/ OXPHOS to impact energy homeostasis on genotoxic stress, steady-state ATP levels did not differ significantly amongst genotypes in unirradiated cells, but were significantly higher $24 \mathrm{~h}$ post irradiation in CX cells (Figure $3 \mathrm{e}$ ), potentially reflecting increased energy production, decreased energy expenditure or both. Taken together, these data support a model in which increased FAO/OXPHOS is a cell-autonomous adaptation to both endogenous and exogenous genotoxic stress in CX cells with the potential to impact energy homeostasis.

Increased FAO is a general response to acute genotoxic stress We next asked if the observed increase in FAO was a specific property of NER deficiency, or a general response to UV-C. As WT cells tolerate the low doses of UV-C used to characterize metabolic changes in CX cells, we exposed WT MDFs and HDFs to higher UV-C doses (20 and $24 \mathrm{~J} / \mathrm{m}^{2}$, respectively) and measured the time-dependent increase in radiolabelled palmitate oxidation. 

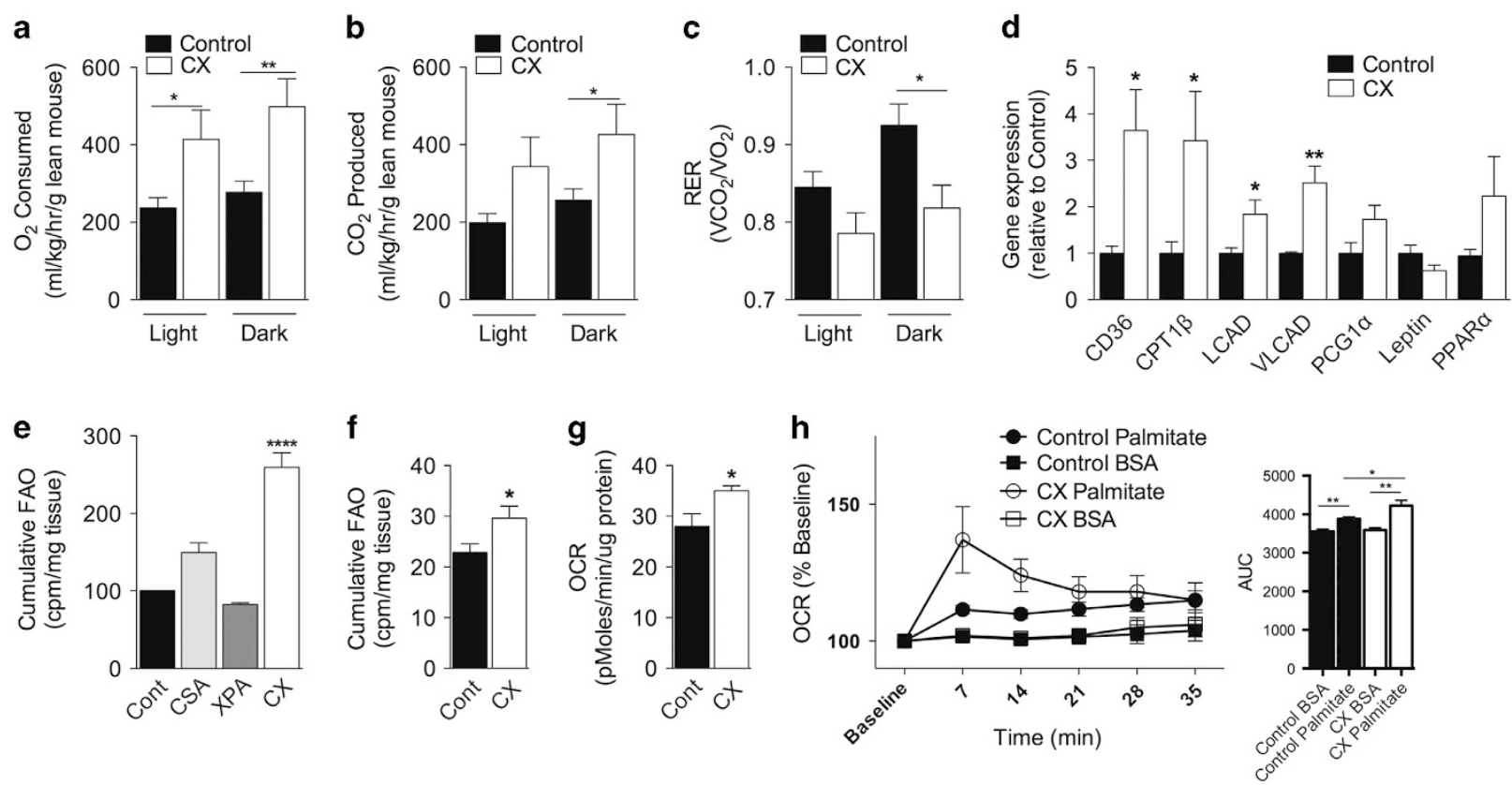

Figure 2. Increased fatty acid oxidation (FAO) in CX mice in vivo and in cells in vitro. (a-c) Indirect calorimetry of 8-14 week control and CX mice ( $n=12$ group) showing $\mathrm{O}_{2}$ consumed (a), $\mathrm{CO}_{2}$ produced (b) and respiratory exchange ratio (RER) (c) all corrected by lean body mass; Student's $t$-test. (d) Gene expression of 12-16 week control and CX muscle ( $n=6 /$ group); Student's $t$-test. (e) Cumulative FAO of tritiated palmitate of soleus muscle from 16 week mice ( $n=5-8 /$ group); 1 -way analysis of variance (ANOVA) with Dunnett's post-test. (f) Cumulative FAO of tritiated palmitate of liver of 16 week mice $(n=5-8 /$ group); Student's $t$-test. (g) Basal oxygen consumption rate (OCR) of mouse dermal fibroblasts (MDFs) measured by Seahorse cell metabolism analyzer in three independent lines of each genotype; Student's $t$-test. (h) OCR expressed as a percentage of baseline within cell line of control and CX MDFs by Seahorse after addition of $200 \mu \mathrm{mol} / \mathrm{l}$ bovine serum albumin (BSA)-conjugated Palmitate with area under the curve anaylsis (AUC) inset at right ( $n=4$ lines/genotype), Student's $t$-test. ${ }^{*} P<0.05,{ }^{*} P<0.01$, $* * * P<.001,{ }^{* * * *} P<0.0001$.

a

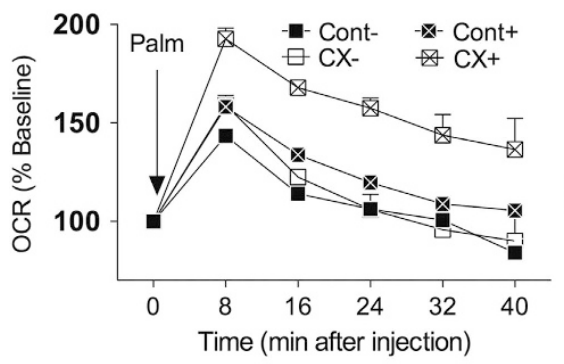

C

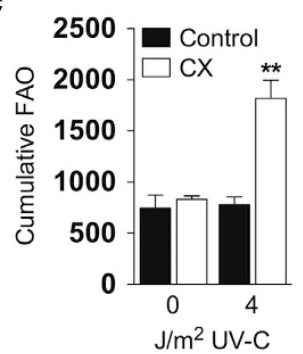

d

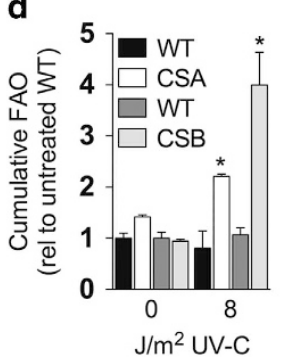

b
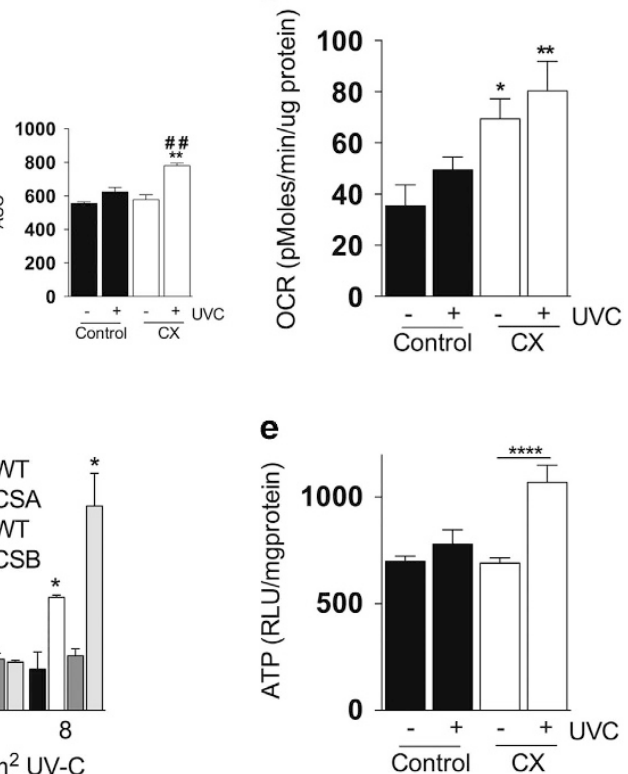

Figure 3. Increased fatty acid oxidation (FAO) is a cell-autonomous, adaptive response triggered by genotoxic stress in CX cells. (a) Oxygen consumption rate (OCR) over time after the injection of $200 \mu \mathrm{mol} / \mathrm{l}$ bovine serum albumin (BSA)-conjugated palmitate in the indicated genotypes ( $n=3$ independent mouse dermal fibroblasts (MDF) lines/genotype) $24 \mathrm{~h}$ post $4 \mathrm{~J} / \mathrm{m}^{2}$ ultraviolet (UV)-C or mock treatment (+ or - , respectively) with AUC analysis at right; Student's $t$-test between genotypes within UV treatment group**; Student's $t$-test within genotype

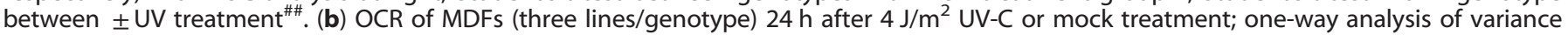
(ANOVA) between genotypes within treatment group with Dunnett's post-test. (c) Cumulative FAO of tritiated palmitate over a $4 \mathrm{~h}$ period, $24 \mathrm{~h}$ after treatment of MDFs ( $n=3$ lines/genotype) with indicated dose of UV-C; Student's $t$-test between genotypes within UV dose. (d) Primary human CSA and CSB dermal fibroblasts treated as in (c); Student's $t$-test between genotypes within UV dose. (e) Steady-state ATP levels of MDFs $24 \mathrm{~h}$ after exposure to 0 or $4 \mathrm{~J} / \mathrm{m}^{2}$ UV-C; Student's $t$-test within genotype between \pm UV treatment. ${ }^{*} P<0.05$, ${ }^{* *} P<0.01$, ${ }^{* * * *} P<0.0001$, $\# \# P<0.01$. 


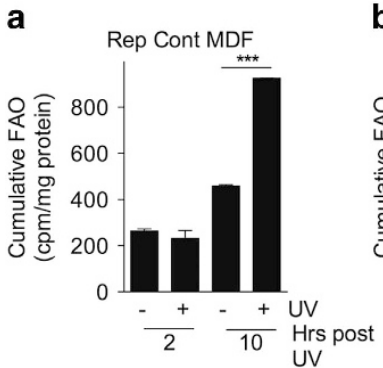

b

e

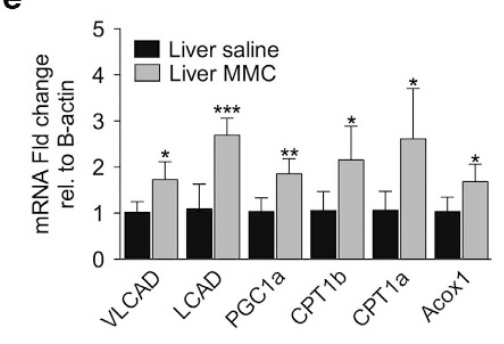

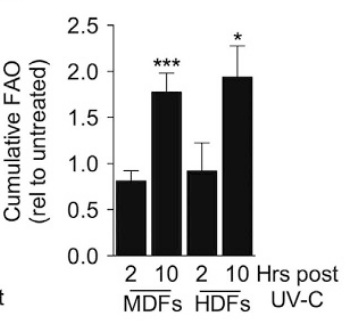

f

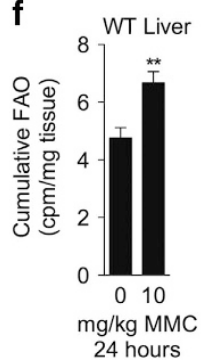

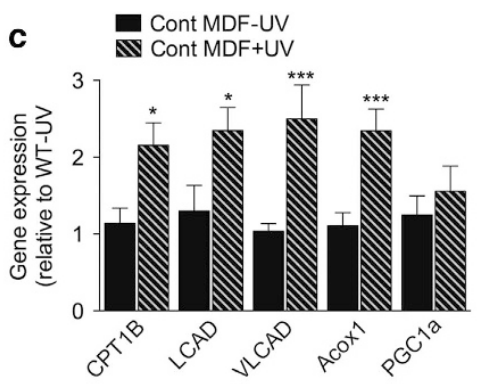

d

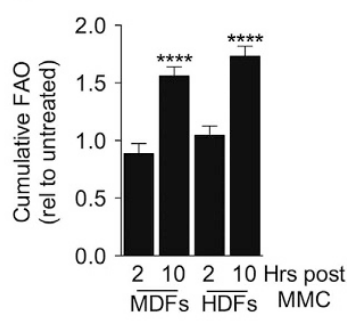

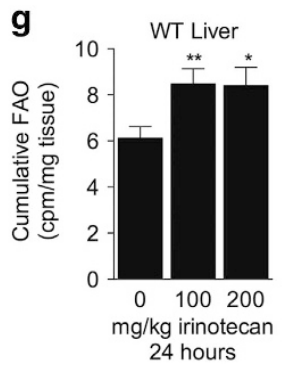

Figure 4. Increased fatty acid oxidation (FAO) is a general response to acute genotoxic stress. (a) Cumulative FAO of tritiated palmitate of a representative control mouse dermal fibroblast (MDF) line (Rep Cont MDF) over the indicated time period after exposure to 0 or $20 \mathrm{~J} / \mathrm{m}^{2} \mathrm{UV}-\mathrm{C}$; Student's $t$-test between treatment groups within time point. (b) Cumulative FAO of tritiated palmitate of two additional UV-C treated control MDF lines expressed relative to untreated at the indicated time point post-irradiation, and of 3 UV-C treated WT human diploid fibroblast (HDF) lines relative to untreated at the indicated time; Student's $t$-test. (c) messenger RNA (mRNA) expression of FAO-related genes in control MDFs $6 \mathrm{~h}$ after exposure to $20 \mathrm{~J} / \mathrm{m}^{2} \mathrm{UV}-\mathrm{C}$ expressed relative to mock irradiated cells; Student's $t$-test. (d) Cumulative FAO of tritiated palmitate of three independent MDF and HDF lines exposed to $40 \mu \mathrm{mol} / \mathrm{l}$ mitomycin C (MMC) expressed relative to untreated at the indicated time point; Student's $t$-test. (e,f) Gene expression of FAO-related genes (e) and FAO capacity (f) in liver from 8 week WT mice injected intraperitoneally (IP) with $10 \mathrm{mg} / \mathrm{kg} \mathrm{MMC} \mathrm{or} \mathrm{saline} \mathrm{and} \mathrm{harvested} \mathrm{after} 24 \mathrm{~h}$ ( $n=4 /$ group); Student's $t$-test between treatment groups. (g) FAO capacity of liver from 8 week WT mice injected IP with 100 or $200 \mathrm{mg} / \mathrm{kg}$ irinotecan or saline and harvested after $24 \mathrm{~h}(n=4 /$ group); Student's $t$-test. * $P<0.05$, ${ }^{* *} P<0.01,{ }^{* * *} P<0.001,{ }^{* * * *} P<0.0001$.

Although no differences in the capacity to burn fatty acid were observed between irradiated and unirradiated MDFs within the first $2 \mathrm{~h}$ after UV-C exposure, by $10 \mathrm{~h}$ irradiated MDFs had oxidized significantly more fatty acid (Figure 4a) despite an overall time-dependent decrease in oxidation rates (Supplementary Figure S4a) likely due to unfavorable culturing conditions (e.g., low glucose, no serum) required for FAO measurements. Additional independent WT MDF and HDF cultures showed similar cumulative increases in FAO between 2 and $10 \mathrm{~h}$ after high-dose UV-C exposure (Figure 4b; Supplementary Figure S4b,c) coincident with an increase in expression of FAO-related genes $6 \mathrm{~h}$ after exposure in WT MDFs (Figure 4c).

Having established that increased FAO occurs upon high doses of UV-C irradiation of WT cells, we next asked if increased FAO is specific to helix-distorting UV lesions or a more general response to genotoxic stress. Treatment with the crosslinking agent MMC resulted in a similar increase in FAO in WT and CX MDFs (Supplementary Figure S4d) as well as in WT MDFs and HDFs (Figure 4d; Supplementary Figure S4e,f). Interestingly, IR, which induces mostly oxidative lesions and some double-stranded DNA breaks, failed to increase FAO capacity in MDFs at doses of 4.6 and $10 \mathrm{~Gy}$, but significantly increased FAO capacity at $100 \mathrm{~Gy}$ (Supplementary Figure S4g,h). All cumulative measurements were normalized to cellular protein in the same well (Supplementary Figure S4i-k).

Finally, we asked if increased FAO is a physiological response to DNA damage in vivo, e.g., in the context of genotoxic chemotherapy. To this end, 9-week-old WT mice were treated with a single chemotherapeutic dose of MMC. One day later, MMC-treated mice displayed significantly increased FAO-related gene expression in liver and muscle (Figure 4e, Supplementary Figure S4I) and an increased FAO capacity in liver ex vivo (Figure 4f). Another chemotherapeutic, irinotecan (topoisomerase I inhibitor), also increased FAO capacity in liver and muscle $24 \mathrm{~h}$ after treatment (Figure 4g, Supplementary Figure S4m), suggesting increased FAO is a general response to genotoxic stress in primary cells in vitro and tissues in vivo.

PARP-1-dependent NAD+/ATP depletion and AMPK activation link DNA damage to increased FAO

How does DNA damage trigger a metabolic switch to increased FAO/OXPHOS? Because FAO provides reducing equivalents for efficient ATP generation via mitochondrial OXPHOS, we hypothesized that the rapid increase in FAO/OXPHOS was an adaptive response to increased energy demands on genotoxic stress. Consistent with this, steady-state ATP levels were transiently reduced in WT MDFs and HDFs within 30-60 min of UV-C or MMC treatment, returning to initial levels within 60-90 min (Figure 5a, Supplementary Figure S5a).

Multiple genotoxins result in activation of PARP- $1,{ }^{13}$ which consumes NAD+ to synthesize PAR chains on target proteins surrounding DNA breaks. The resulting NAD+ depletion can mediate ATP loss through multiple mechanisms, including inhibition of ATP generation by glycolysis for which NAD+ is a key cofactor. ${ }^{12}$ To test the potential of PARP-1 activation to contribute to ATP depletion, we first confirmed its activity by measuring PAR accumulation and NAD+ depletion in WT versus PARP-1 KO primary dermal fibroblasts subject to genotoxic stress. Increased PARylation was observed in the nucleus by immunofluorescence (Supplementary Figure S5b) and in whole cell extracts by western blot (Figure 5b, Supplementary Figure S5c,d) within minutes of MMC or UV-C treatment and coincident with a significant reduction in NAD+ levels (Figure $5 c$ ) in WT but not PARP-1 KO cells.

Importantly, transient ATP depletion and increased FAO capacity observed upon two different forms of genotoxic stress in WT cells were both absent in PARP- 1 KO cells (Figure $5 d, e$, 
a

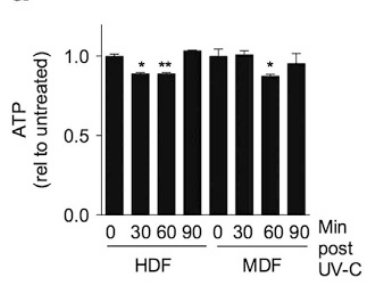

b

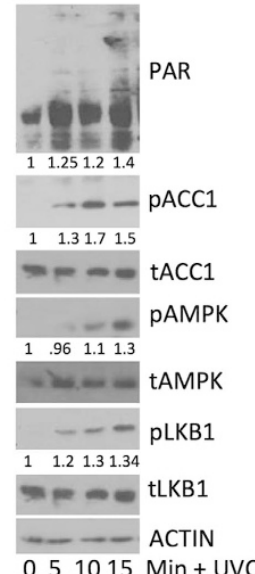

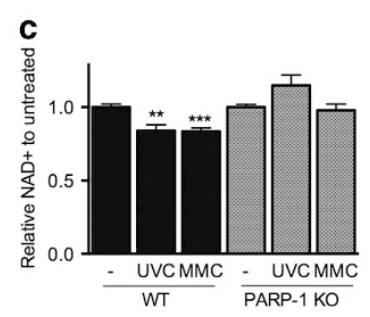

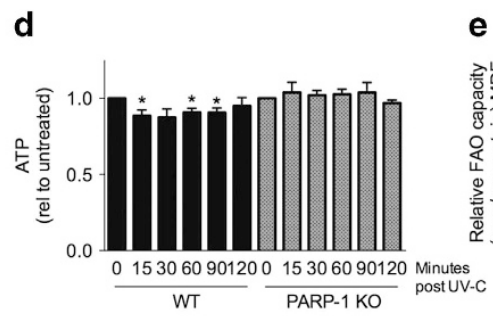

g

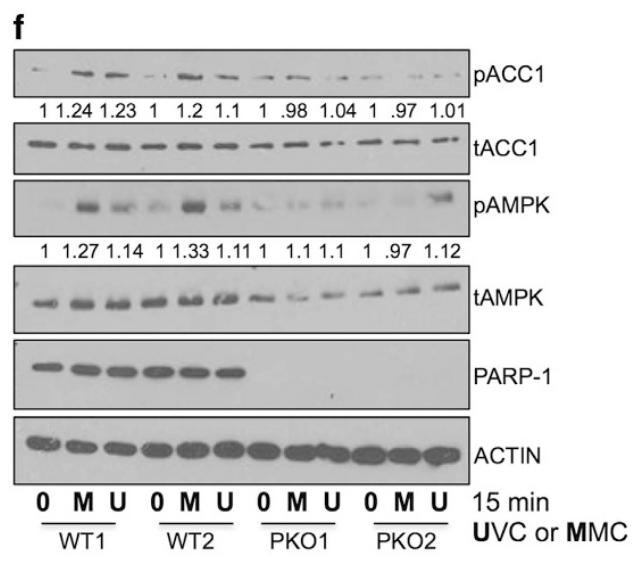

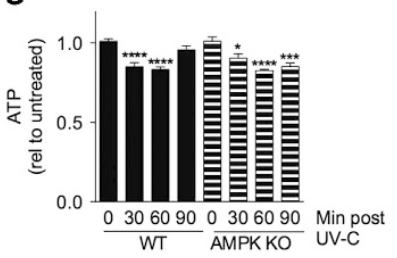

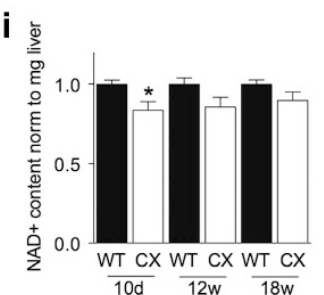

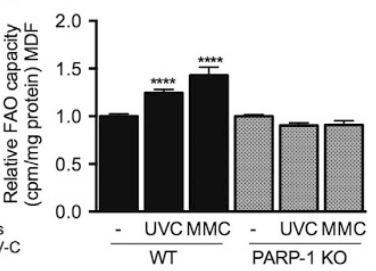

$\mathbf{k}$

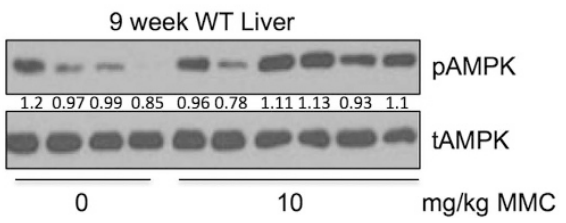

I

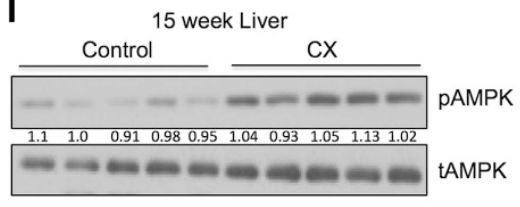

h

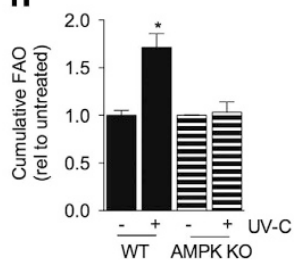

j

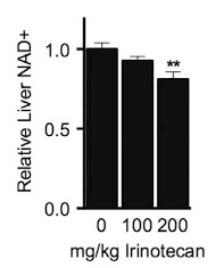

Figure 5. PARP-1-dependent NAD+/ATP depletion and AMPK activation link DNA damage to increased fatty acid oxidation (FAO). (a) Steadystate ATP levels of wild-type (WT) human diploid fibroblasts (HDFs) and mouse dermal fibroblasts (MDFs) after ultraviolet (UV)-C exposure $\left(24 \mathrm{~J} / \mathrm{m}^{2}\right.$ for HDF and $20 \mathrm{~J} / \mathrm{m}^{2}$ for MDFs); one-way analysis of variance (ANOVA) with Dunnett's multiple comparisons test. (b) Western blot of WT MDFs collected 0, 5, 10 and $15 \mathrm{~min}$ after exposure to $20 \mathrm{~J} / \mathrm{m}^{2}$ UV-C. (c) Relative NAD+ content measured in WT and PARP-1 KO MDFs ( $n=4-8$ lines/genotype) $5 \mathrm{~min}$ after treatment with $20 \mathrm{~J} / \mathrm{m}^{2} \mathrm{UV}-\mathrm{C}$ or $40 \mu \mathrm{mol} / \mathrm{l}$ mitomycin C (MMC); Student's $t$-test relative to untreated within genotype. (d) Steady-state ATP levels of WT and PARP-1 KO MDFs ( $n=3-4$ lines/genotype) over a time course after exposure to $20 \mathrm{~J} / \mathrm{m}^{2}$ UV-C; one-way ANOVA with Dunnett's multiple comparisons test. (e) Relative cumulative FAO in WT and PARP-1 KO MDFs $10 \mathrm{~h}$ after exposure to $20 \mathrm{~J} / \mathrm{m}^{2} \mathrm{UV}-\mathrm{C}$ or $40 \mu \mathrm{mol} / \mathrm{I} \mathrm{MMC} \mathrm{(} n=3-4$ lines/genotype); Student's $t$-test relative to untreated within genotype. (f) Western blot of two independent WT and PARP-1 KO MDF lines collected $15 \mathrm{~min}$ after exposure to $\pm 40 \mu \mathrm{mol} / \mathrm{I} \mathrm{MMC} \mathrm{or} 20 \mathrm{~J} / \mathrm{m}^{2}$ UV-C. (g) Steady-state ATP levels of WT and AMPK $\alpha 1$ KO MDFs over a time course after exposure to $20 \mathrm{~J} / \mathrm{m}^{2}$ UV-C ( $n=3-4$ lines/genotype); one-way ANOVA with Dunnett's multiple comparisons test. (h) Relative cumulative FAO in WT and AMPK $\alpha 1$ KO MDFs $10 \mathrm{~h}$ after exposure to $20 \mathrm{~J} / \mathrm{m}^{2} \mathrm{UV}-\mathrm{C}(n=3 \mathrm{lines} /$ genotype); Student's $t$-test relative to untreated within genotype. (i) Relative NAD+ content in livers from WT and CX mice ( $n=3-4 / g e n o t y p e)$ at the indicated ages; Student's $t$-test between genotypes within age. (j) Relative NAD+ content in livers from WT mice intraperitoneally (IP) injected with 100 or $200 \mathrm{mg} / \mathrm{kg}$ irinotecan or saline and harvested after $24 \mathrm{~h}$ ( $n=4 /$ group); Student's $t$-test relative to untreated. (k) Western blot of livers from 8 week WT mice injected IP with MMC or saline and harvested after $24 \mathrm{~h}$ ( $n=4 /$ group);. (I) Western blot of 15 week WT and CX livers. ${ }^{*} P<0.05,{ }^{* *} P<0.01,{ }^{* *} P<0.001,{ }^{* * *} P<0.0001$.

Supplementary Figure S5e,f). These data suggest that PARP-1 may serve as the molecular link between DNA damage and energy metabolism across a spectrum of DNA lesions, likely by binding to a DNA damage repair intermediate common to these different lesions.

AMPK controls metabolic adaptations to ATP depletion including decreased energy expenditure, altered substrate utilization and increased energy efficiency production via OXPHOS. ${ }^{15}$ Consistent with a role for AMPK in metabolic reprogramming in response to DNA damage, MDFs subject to genotoxic stress displayed a rapid, time-dependent increase in phosphorylated/ activated AMPK (Figure 5b) in a PARP-1-dependent manner (Figure 5f, Supplementary Figure S5d).

To test the requirement for AMPK in metabolic reprogramming on genotoxic stress, we used primary dermal fibroblasts isolated from WT and AMPKa1KO mice which lacked detectable expression of the AMPK catalytic subunit (Supplementary Figure S5g). Steadystate ATP levels were reduced in both WT and AMPKa1 KO MDFs in response to $20 \mathrm{~J} / \mathrm{m}^{2} \mathrm{UV}-\mathrm{C}$ or $40 \mu \mathrm{M} \mathrm{MMC}$, but returned to baseline within $2 \mathrm{~h}$ only in WT cells (Figure $5 \mathrm{~g}$, Supplementary Figure S5h), consistent with the inability of AMPKa1 KO cells to maintain energy homeostasis. AMPKa1 KO MDFs also failed to activate $\mathrm{FAO}$ within $10 \mathrm{~h}$ of various genotoxin treatments as in WT cells (Figure 5h, Supplementary Figure S5i,j) without consistent effects on cell viability (Supplementary Figure S5k-m).

To test the potential relevance of PARP-1-mediated NAD+ depletion and AMPK activation in metabolic reprogramming in response to acute or chronic DNA damage in vivo, we analyzed livers from CX versus WT mice at different ages, and from WT mice $24 \mathrm{~h}$ after treatment with a chemotherapeutic genotoxin, irinotecan or MMC. In all cases, we observed reduced steady-state NAD+ levels (Figure $5 i, j$ ) and increased phospho-AMPK (Figure 5k,l). 


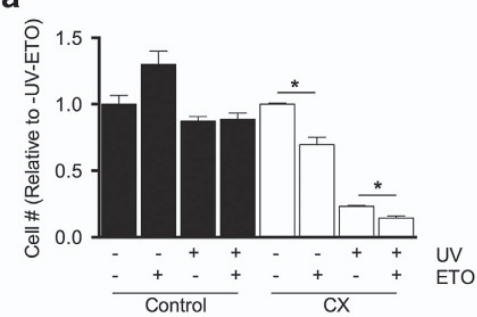

b
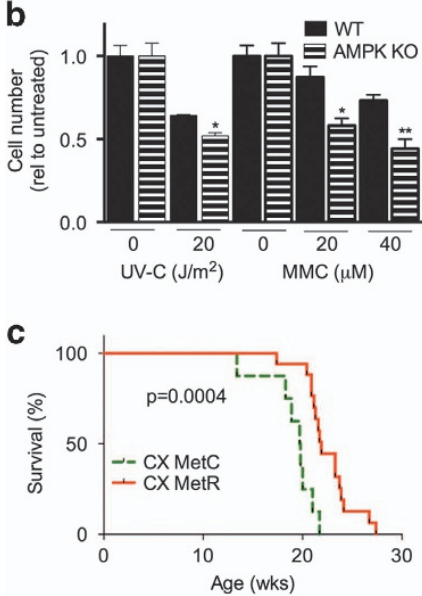

d

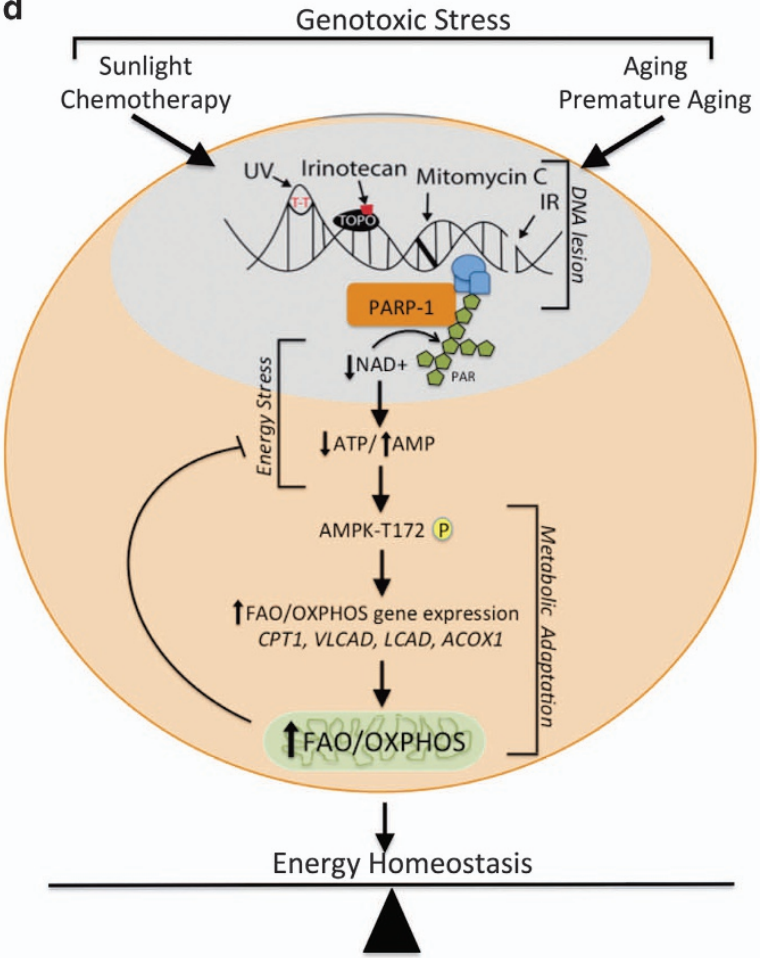

Figure 6. Increased fatty acid oxidation (FAO) is a beneficial adaptive response to genotoxic stress. (a) Relative MDF cell number $24 \mathrm{~h}$ after $20 \mathrm{Jm} /{ }^{2} \mathrm{UV}-\mathrm{C}$ and/or $40 \mu \mathrm{M}$ Etomoxir treatment ( $n=2$ independent MDF lines/genotype in duplicate); Student's $t$-test between indicated groups. (b) Relative cell number of WT and AMPK $\alpha 1$ KO MDFs $24 \mathrm{~h}$ after ultraviolet (UV)-C or mitomycin C (MMC; $n=2 \mathrm{MDF}$ lines/genotype in duplicate); Student's $t$-test versus untreated within genotype and treatment. ${ }^{*} P<0.05$, ${ }^{* *} P<0.01$. (c) Kaplan-Meier survival plots of CX mice on methionine restricted (MetR; $n=17$ ) or methionine complete (MetC; $n=8$ ) diets; log-rank test $P=0.0004$. (d) Model of relationship between DNA damage and maintenance of cellular and organismal energy homeostasis. Acute and chronic genotoxic stress activate PARP-1, depleting steady-state ATP and NAD+ levels, activating AMPK and increasing FAO/OXPHOS to restore energy homeostasis.

Increased FAO is a beneficial adaptive response to genotoxic stress

To determine if increased FAO is a beneficial or maladaptive response to genotoxic stress, we determined cellular hypersensitivity to genotoxic stress in vitro in the presence or absence of the FAO inhibitor, etomoxir. In addition to hypersensitivity to $20 \mathrm{~J} / \mathrm{m}^{2}$ UV-C, CX MDFs displayed reduced viability upon etomoxir treatment in both mock and UV-C treated groups (Figure 6a), consistent with beneficial effects of increased FAO upon both chronic and acute genotoxic stress. A similar hypersensitivity to UV-C and MMC was observed in AMPKa1KO MDFs relative to WT cells (Figure $6 \mathrm{~b}$ ) at doses that increased FAO in WT cells.

Because blocking whole-body FAO in vivo is technically challenging and potentially toxic, we probed the in vivo role of FAO by testing the hypothesis that increased FAO is a beneficial adaptive response to chronic DNA damage in CX mice. To this end, we employed a diet limited for the essential amino acid methionine, which, like DR, promotes a metabolic shift to FAO. ${ }^{26}$ After 2 weeks of methionine restriction (MetR), RER was decreased in CX relative to control mice during the dark phase (Supplementary Figure S6a) without affecting activity (Supplementary Figure S6b) or food intake (Supplementary Figure S6c), consistent with an increase in FAO. Separate cohorts of CX and WT mice on MetR diets from $\sim 6$ weeks also displayed a reduction of body weight (Supplementary Figure S6d) and specifically of the percentage fat mass (Supplementary Figure S6e), with a reciprocal increased percentage lean mass (Supplementary Figure S6f) relative to within genotype controls on the control (MetC) diet. Absolute fat and lean mass also displayed similar trends between genotypes (Supplementary Figure S6g,h). Despite total body fat at or below the level of detection by EchoMRI in the MetR CX group from $\sim 15$ weeks of age, MetR significantly increased median and maximum lifespan of CX mice by $\sim 2$ and 5 weeks, respectively (mean 19.8 weeks MetC versus 21.9 weeks MetR; maximum 21.7 weeks MetC versus 27.4 weeks MetR; log-rank test $P=0.0004$; Figure $6 c$ ). Although the effects of MetR are pleiotropic and extend beyond increased FAO, we can clearly conclude that neither increased FAO nor loss of adiposity exacerbate CX symptoms in vivo; on the contrary, the pro-longevity effects of MetR in this short-lived DNA repair-deficient model are consistent with increased FAO as a beneficial, adaptive response to chronic genotoxic stress in vivo. Taken together, these data support a model in which an AMPK-dependent increase in FAO/OXPHOS is an adaptive response to DNA damage-mediated PARP-1 activation that promotes maintenance of NAD+/ATP energy homeostasis and cell survival (Figure $6 \mathrm{~d}$ ).

\section{DISCUSSION}

Increased FAO/OXPHOS is a cell-autonomous response to DNA damage

Here, we identified a novel link between DNA damage and adaptive cellular/organismal energy metabolism involving an increase in FAO in particular and OXPHOS in general. This connection was observed on acute treatment of WT animals with a variety of genotoxins giving rise to distinct spectrums of DNA lesions, as well as in a model of chronic genotoxic stress induced by congenital DNA repair deficiency. It was also observed upon treatment of different types of primary cells, both human and mouse, with these different genotoxins. Based on these 
considerations, we conclude that this metabolic response represents a general, cell-autonomous response to acute and chronic genotoxic stress.

Why do cells increase OXPHOS on DNA damage, and what is the molecular mechanism linking DNA damage to altered energy metabolism? Our data suggest that multiple different DNA lesions (or potentially common repair intermediates) activate PARP-1, and that PARP-1-dependent activation of AMPK serves as the link between DNA damage and adaptive changes in NAD+ and ATP energy metabolism. Although PARP-1 activation is directly linked to NAD+ depletion, the definitive cause of ATP loss upon PARP-1 activation remains elusive. Several non-mutually exclusive possibilities have been described. NAD+ biosynthesis, either de novo or via the salvage pathway, is an ATP-dependent process, although its contribution to ATP depletion has recently been contested. ${ }^{12}$ Free PAR that is rapidly removed from PARylated proteins can bind to and inhibit hexokinase-1, thus blocking ATP production via glycolysis. ${ }^{12}$ Furthermore, free AMP released upon PAR degradation, e.g., by PARG, could compete with ADP for binding to the adenine nucleotide transporter and further diminish ATP production; consistent with such a possibility, methylnitronitrosoguanidine (MNNG)-induced PAR accumulation and PARG activation results in a time-dependent increase in AMP concentrations and a concomitant decrease in ATP in HeLa cells. ${ }^{27}$ Regardless of the mechanism, each of these could affect ATP levels and thus promote AMPK activation, which contributes to energy homeostasis by blocking anabolic ATP-consuming processes and increasing efficient energy production via mitochondrial OXPHOS, including increased FAO. Although AMPK was previously shown to be activated by phosphorylation on DNA damage, ${ }^{13}$ this is the first demonstration of a role for energy stress itself in DNA damage-related AMPK activation.

It is important to note that while we observed an increase in FAO/OXPHOS as a common response to multiple genotoxic stressors in a variety of primary cells in vitro, as well as in models of acute and chronic DNA damage in vivo, this response was not uniform across doses of all agents. For example, although $100 \mathrm{~Gy}$ IR activated FAO, 5-10 Gy failed to activate FAO despite inducing considerable DNA damage and activating cellular senescence. ${ }^{28}$ This could be explained in part by the previous observation that PARP-1 activation on IR only occurs above $20 \mathrm{~Gy} .{ }^{6}$ Furthermore, low-dose IR ( $<0.1 \mathrm{~Gy}$ ) activates a HIF1a-dependent beneficial adaptive response involving increased glucose uptake and glycolysis in vivo. ${ }^{29}$ DNA damage can also block mitochondrial glutamine anaplerosis via a SIRT4-dependent mechanism, limiting cell proliferation, although whether this has any effect on the balance between oxidative and glycolytic energy metabolism is not known. ${ }^{30}$ Future experiments will be required to determine if and how other forms of DNA damage or DNA repair deficiencies perturb energy metabolism.

Increased FAO/OXPHOS is a beneficial adaptive response to DNA damage

Changes in FAO can be driven either by metabolic inflexibility, e.g., on insulin resistance ${ }^{31}$ or other constitutive defects in glucose metabolic regulation, ${ }^{32}$ or as an adaptive response to environmental cues, ranging from differential substrate availability in response to normal feeding/fasting cycles ${ }^{33}$ or overall restricted food intake. ${ }^{34}$ Our data in the CX model, including improved HOMA-IR in vivo and increased oxygen consumption in cells independent of energy substrate in vitro, are inconsistent with metabolic inflexibility. However, two observations made in this study strongly suggest that increased FAO/OXPHOS is a beneficial adaptive response to genotoxic stress. First, blocking AMPK or FAO reduced cell viability on genotoxic stress; second, increasing FAO extended lifespan in a short-lived mouse model of chronic genotoxic stress.
Interestingly, previous studies on XPA as well as CX mice point to PARP-1 hyperactivation and NAD+ depletion driving mitochondrial dysfunction subsequent to defective SIRT1-dependent mitophagy. ${ }^{35}$ Similalry, age-related accumulation of damaged mitochondria has been observed in the CSB mouse model. ${ }^{36}$ Although these findings may appear at odds with the beneficial metabolic adaptations involving overlapping molecular pathways as described here, they are not necessarily mutually exclusive. We suggest that adaptive changes in energy metabolism may precede constitutive defects in mitochondrial function, but are insufficient to prevent such defects because they fail to address the fundamental defect in DNA repair. Future studies will be required to address this and other possibilities. Nonetheless, our findings have important translational implications for conditions driven by chronic or acute DNA damage as discussed below.

For CS and related progeroid syndromes, these results have implications for our understanding of pleiotropic disease symptoms as well as potential treatment options. Loss of subcutaneous fat is often observed in segmental progeroid disorders such as CS, and has been generally interpreted as a maladaptive response to the rapid aging process. Reduced adiposity and increased FAO in CX mice is unlikely due to any underlying defect in glucose homeostasis, as the response to glucose challenge was normal, and HOMA-IR improved, in CX mice. Instead, our data suggest that loss of adiposity is a constituent of the beneficial adaptive response driven in part by increased fat burning. Consistent with this interpretation, the MetR diet significantly extended longevity of short-lived CX mice despite a further reduction of adiposity. Interestingly, HFD feeding rescues age-dependent hearing loss in a CS model with mild symptoms relative to CX mice. ${ }^{37}$ At face value, beneficial results from varying dietary interventions, HFD and MetR, appears paradoxical. However, under the working assumption that DNA damage-driven energy stress is a major contributor to both hearing loss and reduced lifespan, it then follows that improved maintenance of energy homeostasis either by energy supplementation (HFD) or increased energy efficiency (MetR) could both have beneficial effects. Future experiments will be required to determine if this metabolic adaption to genotoxic stress is present in CS patients and potentially other diseases and their corresponding mouse models with defects in genome maintenance. ${ }^{38}$ However, existing data collectively suggest that strategies to improve energy homeostasis, either by pharmacologic (NAD+ supplementation) or dietary (DR, MetR, ketogenic diet) means, represent exciting new avenues toward mitigation of disease symptoms.

Finally, our findings here have implications for interventional approaches to protect against clinically relevant stressors involving genotoxic stress, ranging from radio/chemotherapeutics to oxidative stress associated with ischemia reperfusion injury. Dietary preconditioning is one such prophylactic approach involving short-term DR or fasting before chemotherapy or surgical stress that significantly reduces detrimental side effects of these interventions; ${ }^{39-41}$ however, the mechanisms of protection remain unclear. Our data suggest the possibility that activation of $\mathrm{FAO} / \mathrm{OXPHOS}$ by diet before genotoxic stress may contribute to protection by preserving energy homeostasis and cell viability, and further that this mechanism may function more effectively when activated before the onset of DNA damage.

\section{MATERIALS AND METHODS}

Mice

All mouse experiments were performed with the approval of the Harvard Medical Area Institutional Animal Care and Use Committee. Mice carrying Xpa and/or Csa knockout alleles in a C57BL/6 background, AMPKa1 (Jackson 014141 bred with E2a-cre) knockout mice in a C57BL/6 background and PARP-1 knockout mice (Jackson 002779) on a 129S background were maintained under standard laboratory conditions 
(temperature $20-24^{\circ} \mathrm{C}$, relative humidity $50-60 \%, 12 \mathrm{~h}$ light $/ 12 \mathrm{~h}$ dark) and allowed free access to food (Research Diets D12450B with 10\% calories from fat, $18 \%$ from casein and $72 \%$ from carbohydrate) and water unless otherwise indicated as described previously. ${ }^{19}$ Other diets utilized include facility chow pellets (PicoLab 5058, Purina), HFD (research diets, 60\% calories from fat), and a 1\% agar-based diet using D12450BSpx (research diets) and individual crystalline amino acids (Ajinomoto) in proportions to those in casein. MetR diets contained $1.5 \mathrm{~g} \mathrm{Met} / \mathrm{kg}$ food and lacking Cys (ref. 42) compared with MetC diet with the same amino acid composition except for $4.5 \mathrm{~g} \mathrm{Met} / \mathrm{kg}$ food. MMC and irinotecan treatment of WT mice was performed by intraperitoneal (IP) injection with $10 \mathrm{mg} / \mathrm{kg}$ MMC or 100/200 mg/kg irinotecan in saline.

\section{Cell lines}

MDFs were passaged in $20 \%$ fetal bovine serum (FBS) in DMEM+Penicillin/ Streptomycin (P/S). Primary human dermal fibroblasts (HDFs) from CS-B patients were a gift from PJ Brooks (CSB: GM00739C and WT: RB4492); CS-A HDFs were purchased from Coriell Cell Repository (CSA: AG10213 and WT: AG10215, AG12726; CSB: AG12724) and passaged in 15\% FBS DMEM+P/S. All cell lines were incubated in $5 \% \mathrm{CO}_{2}$ and $3 \% \mathrm{O}_{2}$. MDFs were treated with 4 or $20 \mathrm{~J} / \mathrm{m}^{2} \mathrm{UV}-\mathrm{C}$ and HDFs at 8 or $24 \mathrm{~J} / \mathrm{m}^{2}$; both were treated with 4.6 , 10 or $100 \mathrm{~Gy} \mathrm{IR,} 40 \mu \mathrm{mol} / \mathrm{I} \mathrm{MMC,} 40 \mu \mathrm{mol} / \mathrm{l}$ compound $C$ and $40 \mu \mathrm{mol} / \mathrm{l}$ Etomoxir (Sigma, St Louis, MO, USA). Cell survival was measured by the relative change in cell number as measured on a hemocytometer following plating of an equal number of cells per line.

\section{Seahorse}

Cellular OCR and ECAR were measured using the Seahorse Cell Metabolism Analyzer XF96. Cells were untreated or irradiated as described above and plated at a density of 18,000 cells/well in a 96-well plate. After $24 \mathrm{~h}$, media changed to unbuffered XF assay media with $11 \mathrm{~m} \mathrm{~mol} / \mathrm{l}$ glucose, $2 \mathrm{~m} \mathrm{~mol} / \mathrm{l}$ glutamine and pyruvate at $\mathrm{pH} 7.4$ and basal OCR and ECAR measured for blocks of 2 min mixing and 5 min measuring. For palmitate assays, cells were treated \pm UV-C, plated at 18,000 cells/96 well and $24 \mathrm{~h}$ later media changed to $\mathrm{KHB}$ buffer $(0.5 \mathrm{~m} \mathrm{~mol} / \mathrm{KCl}, 11.1 \mathrm{~m} \mathrm{~mol} / \mathrm{l} \mathrm{NaCl}, 0.2 \mathrm{~m} \mathrm{~mol} / \mathrm{l}$ $\mathrm{MgSO}_{4}, 0.14 \mathrm{~m} \mathrm{~mol} / \mathrm{l} \mathrm{NaH} \mathrm{PO}_{4}$ and $500 \mu \mathrm{mol} / \mathrm{l}$ Carnitine pH7.4) with $2.5 \mathrm{~m} \mathrm{~mol} / \mathrm{l}$ glucose. After three readings at $2 \mathrm{~min}$ mixing and $5 \mathrm{~min}$ measuring, either bovine serum albumin (BSA) or BSA-conjugated palmitate was injected at $200 \mu \mathrm{mol} / \mathrm{l}$ final concentration, and five more readings recorded. All plates were normalized to protein content as measured after Seahorse by bicinchoninic acid assay (BCA).

\section{Fatty acid oxidation in tissues}

Soleus and liver were removed, weighed and placed in $\mathrm{KH}$ buffer containing $25 \mathrm{~m} \mathrm{~mol} / \mathrm{l} \quad \mathrm{NaHCO}_{3}, 118 \mathrm{~m} \mathrm{~mol} / \mathrm{l} \mathrm{NaCl}, 4.7 \mathrm{~m} \mathrm{~mol} / \mathrm{l} \mathrm{KCl}$, $1.2 \mathrm{~m} \mathrm{~mol} / \mathrm{l} \mathrm{MgSO}, 1.2 \mathrm{~m} \mathrm{~mol} / / \mathrm{NaH}_{2} \mathrm{PO}_{4}, 1.2 \mathrm{~m} \mathrm{~mol} / / \mathrm{CaCl}_{2}$ and glucose $(2.5 \mathrm{~m} \mathrm{~mol} / \mathrm{l})$. The tissues were kept in buffer on ice until all dissections were completed. Each tissue then transferred to $\mathrm{KH}$ buffer plus $2 \%$ fattyacid-free BSA and $2.5 \mathrm{~m} \mathrm{~mol} / \mathrm{I}$ glucose, with $2 \mu \mathrm{Ci}{ }^{3} \mathrm{H}$ palmitic acid and incubated at $37^{\circ} \mathrm{C}$ for $1 \mathrm{~h}$. The buffer was collected and hydrolyzed ${ }^{3} \mathrm{H}$ palmitic acid (as ${ }^{3} \mathrm{H}$ water) was extracted. Buffer $(100 \mu \mathrm{l})$ was added to $100 \mu \mathrm{l}$ of $10 \%$ trichloroacetic acid, vortexed, incubated at RT for 15 min, spun at 16,000 r.p.m. for $10 \mathrm{~min}$, and the supernatant collected into a new tube. Trichloroacetic acid (5\%; $100 \mu \mathrm{l})$ and $40 \mu \mathrm{l} \mathrm{BSA} \mathrm{(10 \% )} \mathrm{was} \mathrm{added} \mathrm{to}$ the supernatant, vortexed, incubated at RT for $15 \mathrm{~min}$, spun at $16,000 \mathrm{rpm}$ for $10 \mathrm{~min}$, and the supernatant transferred to a new tube. Chloroform: methanol $(2: 1,750 \mu l)$ was added to the supernatant, along with $\mathrm{KCl}: \mathrm{HCl}$ $(2 \mathrm{~mol} / \mathrm{l}$ each, $300 \mu \mathrm{l})$, vortexed and spun at 16,000 r.p.m. for $10 \mathrm{~min}$. The upper layer $(\sim 600 \mu \mathrm{l})$ was collected into $5 \mathrm{ml}$ EcoLume, mixed, and counted in a liquid scintillation counter. After subtracting background $\mathrm{cpm}$, the sample cpm was divided by the tissue weight to determine FAO capacity.

\section{Body composition and metabolic parameters}

An EchoMRI device (Echo Medical System, Houston, TX, USA) was used to determine lean and fat mass. Insulin tolerance tests were performed after a $4-5 \mathrm{~h}$ fast by injection of 0.5 units insulin/ $\mathrm{kg}$ body weight into the peritoneum. Glucose tolerance tests were performed after $4-6 \mathrm{~h}$ fast by injection of $2 \mathrm{mg} / \mathrm{kg}$ glucose IP. Blood glucose was measured before and after from tail blood at indicated time points using the OneTouch glucose monitoring system (Lifescan, Milpitas, CA, USA). Serum insulin (Alpco, Salem, NH, USA), leptin (R\&D Systems, Minneapolis, MN, USA) and adiponectin (R\&D Systems) were measured by enzyme-linked immunosorbent assay. Serum triglycerides (Sigma) and beta-hydroxybutyrate (Pointe Scientific, Canton, MI, USA) were measured by enzymatic kits. HOMA-IR was determined using the mouse serum insulin ELISA kit (Alpco).

\section{MDF isolation}

MDFs from pre-weaning WT, CSA, XPA, CX and PARP-1 knockout mice and 20wk WT and AMPKa1KO mice were isolated as described previously. ${ }^{24}$ In brief, tail skin was removed and minced using a razor blade in the presence of $1.6 \mathrm{mg} / \mathrm{ml}$ collagenase II in 20\% FBS DMEM P/S and incubated overnight in one well of a 6 -well plate at $37{ }^{\circ} \mathrm{C}$ in a $5 \% \mathrm{CO}_{2}$ and $3 \% \mathrm{O}_{2}$ incubator. After $\sim 16 \mathrm{~h}$, the minced tissue was pipetted up and down 50 times, passed through a $70 \mu \mathrm{m}$ filter, spun down and resuspended in the above media without collagenase. After a day or two, cells were trypsinized and replated in 2 wells of a 6-well plate. Cells were expanded after culture to $90 \%$ confluency and utilized between passages 3 and 5 .

\section{Indirect calorimetry}

Animals were placed in metabolic cages (Comprehensive Lab Animal Monitoring System (CLAMS), Columbus Instruments, Columbus, OH, USA) for 2 days and data collected from the beginning of the first light cycle. Oxygen consumption and carbon dioxide production were normalized to total lean mouse weight. The RER was determined by the ratio of $\mathrm{CO}_{2}$ produced $\left(\mathrm{VCO}_{2}\right)$ over $\mathrm{O}_{2}$ consumed $\left(\mathrm{VO}_{2}\right)$, both corrected for lean mass. RER values were averaged during the light and dark cycles on a per animal basis. Food consumed was corrected for lean mass of mouse over the 2 day period.

\section{Pre-adipocyte isolation and FACS}

Subcutaneous fat pads from WT, CSA, XPA and CX mice at 14-16 weeks of age were isolated, washed in phosphate-buffered saline (PBS) and minced. Tissue was digested for $60 \mathrm{~min}$ with $1 \mathrm{mg} / \mathrm{ml}$ collagenase II in Hanks' balanced salt solution with $7.5 \%$ FBS then passed through a $70 \mu \mathrm{m}$ filter and spun at $400 \mathrm{~g}$ for $10 \mathrm{~min}$. The floating adipocytes were removed and the pelleted stromal vascular fraction was resuspended in erythrocyte lysis buffer $\left(154 \mathrm{~m} \mathrm{~mol} / \mathrm{l} \mathrm{NH} \mathrm{NH}_{4} \mathrm{Cl} 5.7 \mathrm{~m} \mathrm{~mol} / \mathrm{l} \mathrm{K}_{2} \mathrm{HPO}_{4}, 0.1 \mathrm{~m} \mathrm{~mol} / \mathrm{l}\right.$ EDTA) for $10 \mathrm{~min}$. The cells were then pelleted again, washed in PBS and resuspended in 1:1 DMEM:Ham's F12+P/S and 10\% FBS. Media was changed every $2-3$ days until $90 \%$ confluency was reached in $\sim 7$ days. For FACS analysis, erythrocytes were removed from stromal vascular fraction and 1 million cells were blocked with CD16/32 monoclonal antibody and stained for $30 \mathrm{~min}$ at $4{ }^{\circ} \mathrm{C}$ in the dark with F4/80 FITC and CD11b PeCy7 (at 1:100) all from BioLegend (San Diego, CA, USA). Cells were washed and acquired immediately on a BD FACSCalibur (BD Biosciences, San Jose, CA, USA) and analyzed with FlowJo (Tree Star, Ashland, OR, USA).

\section{Cellular fatty acid oxidation}

Cells were plated at 100,000 cells/well in a 12-well plate in DMEM+P/S with $20 \%$ FBS for MDFs and $15 \%$ for HDFs. For low-dose $\left(4-8 \mathrm{~J} / \mathrm{m}^{2}\right)$ treatments, cells were exposed to UV-C and then returned to standard media for $24 \mathrm{~h}$. For high-dose UV-C (20-24 J/ $\left.\mathrm{m}^{2}\right)$ and IR (4.6-100 Gy) treatments, cells were either exposed, washed with PBS, then incubated for 30 min with DMEM $2 \%$ fatty acid-free BSA and $5.5 \mathrm{~m} \mathrm{~mol} / \mathrm{l}$ glucose or immediately given DMEM $2 \%$ fatty acid-free BSA, and $5.5 \mathrm{~m} \mathrm{~mol} / /$ glucose plus $40 \mu \mathrm{mol} / \mathrm{l}$ MMC for $30 \mathrm{~min}$. Media was then changed to DMEM $2 \%$ fatty acid-free BSA, $5.5 \mathrm{~m} \mathrm{~mol} / \mathrm{l}$ glucose ( \pm genotoxin), and $2 \mu \mathrm{Ci}^{3} \mathrm{H}$ palmitic acid and incubated for 2-10 h. The media was then collected and hydrolyzed ${ }^{3} \mathrm{H}$ palmitic acid (as ${ }^{3} \mathrm{H}$ water) was extracted by the same technique as in tissues. After the media was removed, the cells were washed with $1 \times$ PBS and incubated for 10 min with $0.1 \mathrm{M} \mathrm{NaOH}$ at room temperature, and total protein collected for quantitation using BSA standards. After subtracting the background cpm, the sample cpm was divided by the protein concentration for a measure of cumulative FAO.

\section{ATP measurements}

MDFs and HDFs were plated at a density of 10,000 cells per 96 well. Sixteen hours later, cells were dosed with UV-C, IR or $40 \mu \mathrm{mol} / \mathrm{I} \mathrm{MMC.} \mathrm{Up} \mathrm{to} 2 \mathrm{~h}$ later, $100 \mu$ l fresh media was added and ATP measured on the addition of $100 \mu \mathrm{l}$ Cell Titer Glo reagent (Promega, Madison, WI, USA). The plate was shaken for $2 \mathrm{~min}$ and then incubated at room temperature in the dark for $10 \mathrm{~min}$ before determining the luminescence in a plate reader (Biotek 
Synergy 2). ATP content was normalized to protein content as determined by BCA.

\section{Mitochondrial content}

$18,000 \mathrm{MDF}$ were plated after 0 or $4 \mathrm{~J} / \mathrm{m}^{2} \mathrm{UV}-\mathrm{C}$ treatment and placed at $37^{\circ} \mathrm{C}$ in a $5 \% \mathrm{CO}_{2}, 3 \% \mathrm{O}_{2}$ incubator for $24 \mathrm{~h}$. Media was then changed to $80 \mathrm{nM}$ Mitoview Green in standard MDF media for $30 \mathrm{~min}$. Cells were washed with PBS and read on a plate reader.

\section{Quantitative real-time PCR}

Total RNA was isolated from cells and tissues using Qiazol (Qiagen, Hilden, Germany) and complementary DNA synthesized by random hexamer priming with the Verso CDNA kit (Thermo Fisher, Waltham, MA, USA). quantitative real-time PCR was performed using Taq-Pro DNA polymerase (Denville Scientific, Holliston, MA, USA) and SYBR green dye (Lonza, Portsmouth, NH, USA). Fold changes were calculated by the $\Delta \Delta C_{t}$ method using B-actin as a standard, and normalized to the experimental WT control. Primer sequences are as follows: B-actin F: 5'-AGCTTCTTTGCAGCTC CTTCGTTG R: TTCTGACCCATTCCCACCATCACA-3'; CD36; F: 5'-GAGCAACTGG TGGATGGTIT R: GCAGAATCAAGGGAGAGCAC-3'; CPT1 $\beta$ F: 5'-TTGCCCTACAG CTGGCTCATTTCC R: GCACCCAGATGATTGGGATACTGT-3'; LCAD F:5'-TCTITC CTCGGAGCATGACA R: GACCTCTCTACTCACTTCTCCAG-3'; VLCAD F:5'-CTACTG TGCTTCAGGGACACC R: CAAAGGACTTCGATTCTGCCC-3'; PGC1 a F: 5'-AGCCGT GACCACTGACAACGAG R: GCTGCATGGTTCTGAGTGCTAAG-3'; Leptin F: 5'-TG AAGCCCAGGAATGAAGTC R: TCAAGACCATTGTCACCAGG-3'; PPARa F: 5'-TGTTTGTGGCTGCTATAATTTGC; R: GCAACTTCTCAATGTAGCCTATGTTT-3'; Acox1 F: 5'-CCTGATTCAGCAAGGTAGGG R: TCGCAGACCCTGAAGAAATC-3'.

\section{Histology}

Perigonadal white adipose tissue (WAT) depots were isolated, washed in PBS and fixed in Bouin's solution for $24 \mathrm{~h}$. Paraffin-embedded tissue was then sectioned and stained with hematoxylin and eosin for overall morphology or immunostained as previously described using rat antimouse F4/80 diluted 1:200 (eBioscience, San Diego, CA, USA). Controls were performed simultaneously.

\section{Westerns}

Tissues and cells were homogenized with NP-40 buffer containing protease and phosphatase inhibitors and dithiothreitol (DTT). Samples were normalized for protein content, boiled with sodium dodecyl sulfate loading buffer separated by sodium dodecyl sulfate-polyacrylamide gel electrophoresis, transferred to polyvinylidene difluoride membrane (Whatman, Maidstone, UK) and blotted for phospho-ACC1 (S79, \#3661), phospho-AMPK (T172, \#2535), ACC1 (\#4190), AMPK (\#2532), phospho-LKB1 (\#3482), LKB1 (\#3050), Parp-1 (\#9542) and Actin (\#4967) from Cell Signaling Technologies (Danvers, MA, USA) and PAR (51-811HKC) from BD Biosciences (San Jose, CA, USA).

\section{Lipid profiling}

Sera lipidomics profiling was conducted using liquid chromatography separations coupled with mass spectrometry based analysis on a Thermo Exactive Mass Spectrometer. Detailed methods have been described. ${ }^{43,44}$

\section{NAD+ determination}

MDFs were plated at 500,000 per $10 \mathrm{~cm}$ plate. The following day media was removed, cells treated $\pm 20 \mathrm{~J} / \mathrm{m}^{2} \mathrm{UV}-\mathrm{C}$, and then media returned. Five minutes later, cells were washed with PBS, trypsinized and NAD+ determined using the EnzyChrom NAD+/NADH Assay kit (E2ND-100, Bio-Assay systems). For tissues, approximately $20 \mathrm{mg}$ size pieces were prepared and used in the same assay and normalized to protein content by BCA.

\section{Immunofluorescence}

MDFs were plated on $18 \mathrm{~mm}$ circular coverslips at 350,000 cells per well in a 12-well plate. Cells were treated with $40 \mu \mathrm{mol} / \mathrm{IMMC}$ or $20 \mathrm{~J} / \mathrm{m}^{2} \mathrm{UV}-\mathrm{C}$, incubated for the indicated amount of time, rinsed twice with PBS and permeabilized $5 \mathrm{~min}$ at room temperature with $0.5 \%$ Triton in PBS. Cells were then fixed in $3.7 \%$ formaldehyde in PBS for 10 min and washed twice in PBS. Cells were then incubated for $10 \mathrm{~min}$ in $0.5 \% \mathrm{NP}-40$ in PBS, washed in PBS, then blocked in PBS+0.5\% BSA (PBB) for 20 min. Primary antibody
(1:200, anti-PAR clone 10H, Enzo Life Sciences, Farmingdale, NY, USA) was incubated in PBB overnight at $4{ }^{\circ} \mathrm{C}$. Cells were then washed $3 \times 5 \mathrm{~min}$ in PBB, incubated $1 \mathrm{~h}$ at $37^{\circ} \mathrm{C}$ in secondary (goat anti-mouse 1:1000 Alexa Fluor Thermo Fisher) in PBB. Cells were washed $3 \times 5$ min in PBB, rinsed in PBS, and mounted with 4,6-diamidino-2-phenylindole on slides. Cells were imaged using a Zeiss LSM 700 confocal microscope, acquired using Zen Black software and analyzed using Fiji software.

\section{Statistics}

The indicated statistical analyses were performed either in Excel or in GraphPad Prism.

\section{ACKNOWLEDGEMENTS}

We thank David Sinclair and Zhi-Min Yuan for insightful discussions; Gokhan Hotamisligil for use of metabolic facilities; PJ Brooks for sharing cell lines; Casimiro Gerarduzzi for assistance with IR procedures; and Brien Hopkins for graphical assistance. This work was funded in part by grants from the Luke O'Brien Foundation, NIA AG-036712, and NIDDK DK-090629 to JRM, P30-DK040561 to BSK, Swiss National Science Foundation P1LAP3 158895 to $\mathrm{AL}, \mathrm{NIH}$ training grants (Interdisciplinary Genes and Environment T32ES016645, Radiation Biology T32CA009078), and the National Science Foundation Graduate Research Fellowship NSF-DGE1144152 to LEB.

\section{CONTRIBUTIONS}

LEB, SCV and JRM designed the experiments. LEB, SCV, KS, RMG, AL, JHTV, PM, DV and $\mathrm{KI}$ performed the experiments. LEB, VRM, RTB, C-HL, EN, BSK and JRM analyzed the data. LEB and JRM wrote the paper. All authors reviewed the paper.

\section{COMPETING INTERESTS}

BSK is the inventor on general metabolomics-related IP that has been licensed to Metabolon via Weill Medical College of Cornell University and for which he receives royalty payments via Weill Medical College of Cornell University. He also consults for and has a small equity in the company. Metabolon offers biochemical profiling services and is developing molecular diagnostic assays detecting and monitoring disease. Metabolon has no rights or proprietary access to the research results presented and/or new IP generated under these grants/studies. BSK's interests were reviewed by the Brigham and Women's Hospital and Partners Healthcare in accordance with their institutional policy. Accordingly, upon review, the institution determined that BSK's financial interest in Metabolon does not create a significant financial conflict of interest with this research. The addition of this statement where appropriate was explicitly requested and approved by $\mathrm{BWH}$.

\section{REFERENCES}

1. Marczuk-Krynicka, D., Hryniewiecki, T., Piatek, J. \& Paluszak, J. The effect of brief food withdrawal on the level of free radicals and other parameters of oxidative status in the liver. Med. Sci. Monit. 9, BR131-BR135 (2003).

2. Taguchi, K., Motohashi, H. \& Yamamoto, M. Molecular mechanisms of the Keap1Nrf2 pathway in stress response and cancer evolution. Genes Cells 16, 123-140 (2011).

3. Bhatt, A. N. et al. Transient elevation of glycolysis confers radio-resistance by facilitating DNA repair in cells. BMC Cancer 15, 335 (2015).

4. Fox, C. J., Hammerman, P. S. \& Thompson, C. B. Fuel feeds function: energy metabolism and the T-cell response. Nat. Rev. Immunol. 5, 844-852 (2005).

5. Jacobson, E. L., Antol, K. M., Juarez-Salinas, H. \& Jacobson, M. K. Poly(ADP-ribose) metabolism in ultraviolet irradiated human fibroblasts. J. Biol. Chem. 258, 103-107 (1983).

6. Oleinick, N. L. \& Evans, H. H. Poly(ADP-ribose) and the response of cells to ionizing radiation. Radiat. Res. 101, 29-46 (1985).

7. Bowman, K. J., Newell, D. R., Calvert, A. H. \& Curtin, N. J. Differential effects of the poly (ADP-ribose) polymerase (PARP) inhibitor NU1025 on topoisomerase I and II inhibitor cytotoxicity in L1210 cells in vitro. Br. J. Cancer 84, 106-112 (2001).

8. Whish, W. J., Davies, M. I. \& Shall, S. Stimulation of poly(ADP-ribose) polymerase activity by the anti-tumour antibiotic, streptozotocin. Biochem. Biophys. Res. Commun. 65, 722-730 (1975).

9. Berger, N. A. Poly(ADP-ribose) in the cellular response to DNA damage. Radiat. Res. 101, 4-15 (1985).

10. Alano C. C. et al. NAD+ depletion is necessary and sufficient for poly(ADP-ribose) polymerase-1-mediated neuronal death. J. Neurosci. 30, 2967-2978 (2010).

11. Sims, J. L., Berger, S. J. \& Berger, N. A. Poly(ADP-ribose) Polymerase inhibitors preserve nicotinamide adenine dinucleotide and adenosine 5 '-triphosphate 
pools in DNA-damaged cells: mechanism of stimulation of unscheduled DNA synthesis. Biochemistry 22, 5188-5194 (1983).

12. Fouquerel, E. et al. ARTD1/PARP1 negatively regulates glycolysis by inhibiting hexokinase 1 independent of NAD+ depletion. Cell Rep. 8, 1819-1831 (2014).

13. Bungard, D. et al. Signaling kinase AMPK activates stress-promoted transcription via histone H2B phosphorylation. Science 329, 1201-1205 (2010).

14. Kim, S. Y. et al. AMP-activated protein kinase-alpha1 as an activating kinase of TGF-beta-activated kinase 1 has a key role in inflammatory signals. Cell Death Dis. 3, e357 (2012).

15. Hardie, D. G., Ross, F. A. \& Hawley, S. A. AMPK: a nutrient and energy sensor that maintains energy homeostasis. Nat. Rev. Mol. Cell Biol. 13, 251-262 (2012).

16. Budanov, A. V. \& Karin, M. p53 target genes sestrin 1 and sestrin2 connect genotoxic stress and mTOR signaling. Cell 134, 451-460 (2008).

17. Natale, V. A comprehensive description of the severity groups in Cockayne syndrome. Am. J. Med. Genet. A 155A, 1081-1095 (2011).

18. Sykora, P., Wilson, D. M. 3rd \& Bohr, V. A. Repair of persistent strand breaks in the mitochondrial genome. Mech. Ageing Dev. 133, 169-175 (2012).

19. Brace L. E. et al. Lifespan extension by dietary intervention in a mouse model of Cockayne syndrome uncouples early postnatal development from segmental progeria. Aging Cell 12, 1144-1147 (2013).

20. van de Ven, M. et al. Extended longevity mechanisms in short-lived progeroid mice: identification of a preservative stress response associated with successful aging. Mech. Ageing Dev. 128, 58-63 (2007).

21. Guo, Y. et al. Pancreatic islet-specific expression of an insulin-like growth factortransgene compensates islet cell growth in growth hormone receptor genedeficient mice. Endocrinology 146, 2602-2609 (2005).

22. Bruss, M. D., Khambatta, C. F., Ruby, M. A., Aggarwal, I. \& Hellerstein, M. K. Calorie restriction increases fatty acid synthesis and whole body fat oxidation rates. Am. J. Physiol. Endocrinol. Metab. 298, E108-E116 (2010).

23. Susa, D. et al. Congenital DNA repair deficiency results in protection against renal ischemia reperfusion injury in mice. Aging Cell 8, 192-200 (2009).

24. van de Ven, M. et al. Adaptive stress response in segmental progeria resembles long-lived dwarfism and calorie restriction in mice. PLoS Genet. 2, e192 (2006).

25. Wang, Y. et al. Dysregulation of gene expression as a cause of Cockayne syndrome neurological disease. Proc. Natl Acad. Sci. USA 111, 14454-14459 (2014).

26. Hasek, B. E. et al. Dietary methionine restriction enhances metabolic flexibility and increases uncoupled respiration in both fed and fasted states. Am. J. Physiol. Regul. Integr. Comp. Physiol. 299, R728-R739 (2010).

27. Formentini, L. et al. Poly(ADP-ribose) catabolism triggers AMP-dependent mitochondrial energy failure. J. Biol. Chem. 284, 17668-17676 (2009).

28. Rodier, F. et al. Persistent DNA damage signalling triggers senescence-associated inflammatory cytokine secretion. Nat. Cell Biol. 11, 973-979 (2009).

29. Lall, R. et al. Low-dose radiation exposure induces a HIF-1-mediated adaptive and protective metabolic response. Cell Death Differ. 21, 836-844 (2014).

30. Jeong, S. M. et al. SIRT4 has tumor-suppressive activity and regulates the cellular metabolic response to DNA damage by inhibiting mitochondrial glutamine metabolism. Cancer Cell 23, 450-463 (2013).

31. Hancock, C. R. et al. High-fat diets cause insulin resistance despite an increase in muscle mitochondria. Proc. Natl Acad. Sci. USA 105, 7815-7820 (2008).
32. Zhang, S., Hulver, M. W., McMillan, R. P., Cline, M. A. \& Gilbert, E. R. The pivotal role of pyruvate dehydrogenase kinases in metabolic flexibility. Nutr Metab (Lond) 11, 10 (2014).

33. Hue, L. \& Taegtmeyer, H. The Randle cycle revisited: a new head for an old hat Am. J. Physiol. Endocrinol. Metab. 297, E578-E591 (2009).

34. Cao, S. X., Dhahbi, J. M., Mote, P. L. \& Spindler, S. R. Genomic profiling of shortand long-term caloric restriction effects in the liver of aging mice. Proc. Natl Acad. Sci. USA 98, 10630-10635 (2001).

35. Fang, E. F. et al. Defective mitophagy in XPA via PARP-1 hyperactivation and NAD (+)/SIRT1 reduction. Cell 157, 882-896 (2014).

36. Scheibye-Knudsen, M. et al. Cockayne syndrome group B protein prevents the accumulation of damaged mitochondria by promoting mitochondrial autophagy. J. Exp. Med. 209, 855-869 (2012).

37. Scheibye-Knudsen, M. et al. A High-Fat Diet and NAD(+) Activate Sirt1 to Rescue Premature Aging in Cockayne Syndrome. Cell Metab. 20, 840-855 (2014).

38. Hasty, P., Campisi, J., Hoeijmakers, J., van Steeg, H. \& Vijg, J. Aging and genome maintenance: lessons from the mouse? Science 299, 1355-1359 (2003).

39. Lee, C. et al. Fasting cycles retard growth of tumors and sensitize a range of cancer cell types to chemotherapy. Science translational medicine 4 124ra127 (2012).

40. Peng, W. et al. Surgical stress resistance induced by single amino acid deprivation requires Gcn2 in mice. Sci. Transl. Med. 4, 118ra111 (2012).

41. Mitchell, J. R. et al. Short-term dietary restriction and fasting precondition against ischemia reperfusion injury in mice. Aging Cell. 9, 40-53 (2010).

42. Miller R. A. et al. Methionine-deficient diet extends mouse lifespan, slows immune and lens aging, alters glucose, T4, IGF-I and insulin levels, and increases hepatocyte MIF levels and stress resistance. Aging Cell 4, 119-125 (2005).

43. Bird, S. S., Marur, V. R., Sniatynski, M. J., Greenberg, H. K. \& Kristal, B. S. Serum lipidomics profiling using LC-MS and high-energy collisional dissociation fragmentation: focus on triglyceride detection and characterization. Anal. Chem. 83, 6648-6657 (2011).

44. Bird, S. S., Marur, V. R., Sniatynski, M. J., Greenberg, H. K. \& Kristal, B. S. Lipidomics profiling by high-resolution LC-MS and high-energy collisional dissociation fragmentation: focus on characterization of mitochondrial cardiolipins and monolysocardiolipins. Anal. Chem. 83, 940-949 (2011).

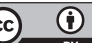

This work is licensed under a Creative Commons Attribution 4.0 International License. The images or other third party material in this article are included in the article's Creative Commons license, unless indicated otherwise in the credit line; if the material is not included under the Creative Commons license, users will need to obtain permission from the license holder to reproduce the material. To view a copy of this license, visit http://creativecommons.org/licenses/ by/4.0/

(c) The Author(s) 2016

Supplementary Information accompanies the paper on the npj Aging and Mechanisms of Disease website (http://www.nature.com/npjamd) 